\title{
Laparoscopic conservative surgery of colovesical fistula: is it the right way?
}

\author{
Giovanni Cochetti ${ }^{1}$, Emanuele Cottini ${ }^{1}$, Roberto Cirocchi ${ }^{2}$, Alberto Pansadoro ${ }^{1}$, Emanuele Lepri ${ }^{1}$, Alessia Corsi ${ }^{2}$, \\ Francesco Barillaro ${ }^{1}$, Ettore Mearini ${ }^{1}$ \\ 1Department of Surgical Specialties, Urological Andrological Surgery and Minimally Invasive Techniques, University of Perugia, Terni, Italy \\ 2Department of Surgery, University of Perugia, Italy
}

Videosurgery Miniinv 2013; 8 (2): 162-165 DOI: $10.5114 /$ wiitm.2011.32808

\begin{abstract}
Enterovesical fistula is a rare disease. The standard treatment of colovesical fistula is removal of the fistula, suture of the bladder wall, and colic resection with or without temporary colostomy. The usual approach is open because the laparoscopic one has high conversion rates and morbidity. We report the first laparoscopic conservative treatment of colovesical fistula in our knowledge and its long-term results. A 69-year-old man was affected by colovesical fistula due to endoscopic exeresis of a $2 \mathrm{~cm}$ adenomatous polyp in the sigmoid diverticulum. We performed a laparoscopic conservative treatment of the fistula without colic resection. Operative time was $210 \mathrm{~min}$ and estimated blood loss was $300 \mathrm{ml}$. The catheter was removed after 10 days. Time to first flatus was 2 days and the hospital stay was 8 days. No peri- or post-operative complications occurred. At 48-month follow-up fistula did not recur. Laparoscopic conservative surgery for colovesical fistula is safe and feasible. It could be a therapeutic option in selected cases, especially if diverticular disease and inflammation are slight.
\end{abstract}

Key words: colovesical fistula, laparoscopic conservative treatment, colon resection, diverticular disease.

\section{Introduction}

Enterovesical fistula is a rare disease that affects more males than females with a ratio of $3: 1$. This is because the presence of the uterus between the bladder and the colon-rectum seems to reduce the risk of enterovesical fistula [1, 2]. Enterovesical fistula can be classified according to the involved intestinal segment: colovesical, rectovesical, ileovesical and appendicovesical. Colovesical fistula is the most frequent and usually it develops between the sigmoid colon and dome of the bladder. Colovesical fistula represents about $70 \%$ of all fistulas, ileovesical one $15 \%$, rectovesical one $10 \%$, appendicovesical one 5\% [1]. Forty - eighty-eight percent of colovesical fistulas are due to complicated diverticu- lar disease [3]. About $80-85 \%$ of patients with diverticular disease remain asymptomatic, while 15\% develop symptomatic diverticular disease. However, these patients have symptoms but no inflammatory signs [4]. About $5 \%$ of patients with diverticula develop diverticulitis and only $2 \%$ develop complications of diverticulitis such as abscess, fistula, obstruction or haemorrhage [5]. Fistula arising from diverticulitis is due to the inflammation of tissues surrounding the bladder. Sometimes it could result from a diverticular abscess that undermines the bladder wall, developing a colovesical fistula. Other causes are colorectal cancer, Crohn's disease, iatrogenic and traumatic causes [3]. The diagnosis is clinical. Pneumaturia (67-85\%) and faecaluria (45-65\%) are con- 
sidered pathognomonic. Recurrent genito-urinary infections occur in $57 \%$ of cases and generally they are due to the typical bacteria of intestinal flora [6]. Recurrent diverticulitis often occurs before urinary infections. The study of the urinary tract with urinalysis, urine culture and cystoscopy and the study of the intestinal tract with computed tomography (CT) scan, Barium enema and colonoscopy are helpful in order to plan the best surgical approach. The standard treatment of colovesical fistula is exeresis of the fistula following suture of the bladder wall and colic resection with or without temporary colostomy. The conservative treatment does not include colon resection. Laparoscopy is not considered as first choice treatment and usually the approach is open [7].

We report the first laparoscopic conservative treatment of colovesical fistula as our knowledge and its long-term results.

\section{Case report}

A 69-year-old man presented with recurrent cystitis with fever and pneumaturia that appeared after endoscopic removal of a $2 \mathrm{~cm}$ adenomatous polyp in the sigmoid diverticulum. Cystoscopy showed bullous oedema surrounding the erythematous area in the bladder. The biopsies of this area detected urothelial hyperplasia and chronic inflammation. Computed tomography scan showed air/fluid level in the bladder, slight anomalies and thickening of the bladder wall which was fixed to the sigmoid wall; no fistula was evident. Colonoscopy confirmed a small sigmoid diverticulum without any fistula.

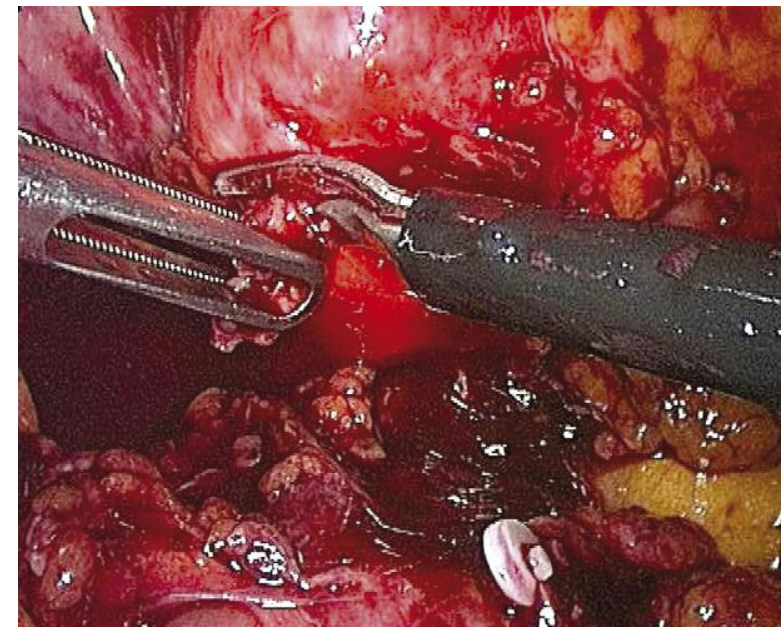

Photo 1. The fistulous tract was clipped
We planned laparoscopic abdominal exploration: a $12 \mathrm{~mm}$ trocar for the camera was placed at the umbilicus, two $10 \mathrm{~mm}$ trocars were placed at the level of the right and left iliac fossa and an assistant $5 \mathrm{~mm}$ port was placed along the left mid-clavicular line. We detected a fistulous tract between the sigmoid colon and bladder that was carefully dissected. Since diverticular disease was slight, we decided not to perform colon resection. We clipped the fistulous loop with weck ${ }^{\circledR} \mathrm{Hem}-$ o-lock ${ }^{\circledR}$ clips and then we removed it (Photos 1 and 2). Thus we divided the bladder wall from the sigma. We sutured and sinked the sigmoid wall and performed a curettage of the fistula site. The bladder wall was closed with a single layer running suture using Vicryl 2-0. We placed fatty tissue between the sigmoid and bladder wall in order to reduce the risk of recurrence of the fistula. The follow up was 48 months.

Operative time was $210 \mathrm{~min}$ and estimated blood loss was $300 \mathrm{ml}$. Ten days later a voiding cystourethrogram showed absence of leakage and the catheter was removed (Photo 3). The post-operative pain was low and the VAS scale score was 2 . Time to first flatus was 2 days and the hospital stay was 8 days. The histopathological examination of the fistula showed colonic inflammatory tissue and necrotic tissue. No peri- or post-operative complications or fistula recurrence occurred at 48-month follow-up.

\section{Discussion}

The issue of colovesical fistula treatment remains controversial. It has not yet been established which is

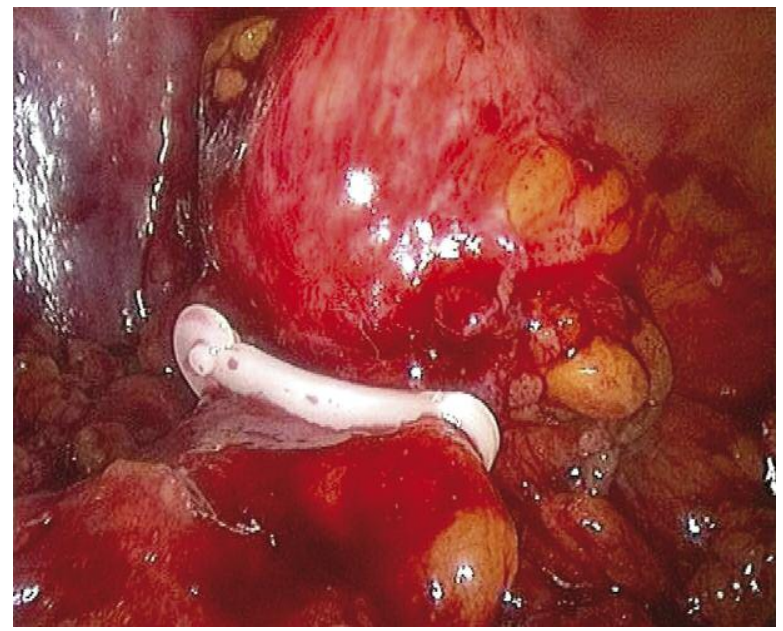

Photo 2. The fistulous tract was removed 


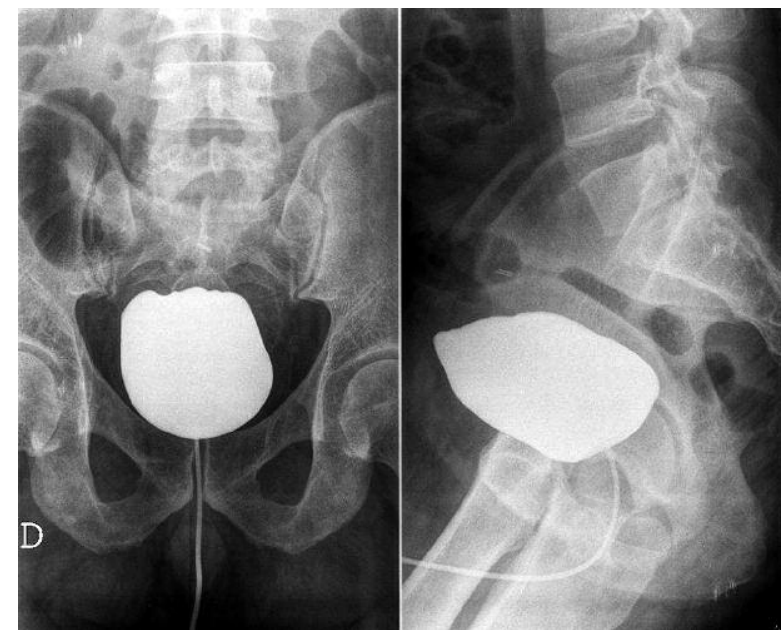

Photo 3. Post-operative cystourethrogram shows absence of leakage

the best therapeutic approach when we suspect a colovesical fistula. Also the American Society of Colon and Rectal Surgeons' Task Force guidelines on sigmoid diverticulitis do not state the most appropriate way of treatment for colovesical fistula.

Historically, fistulas that complicated diverticular disease were treated with a preliminary colostomy, resection and final colostomy closure in a three-stage procedure. In 1950, Charles W. Mayo proposed the one-stage resection. To date the standard treatment of colovesical fistula is removal of the fistula, suture of the bladder wall after curettage or excision of the bladder cuff, and colic resection with one or two stage anastomosis [7]. Commonly an omental flap is placed between the bladder and colon in order to minimize recurrence rates [8]. Usually the approach is open, because the laparoscopic one is burdened by a high conversion rate and morbidity [9]. Only a few cases of laparoscopic treatment of diverticulitis fistula are reported in the literature. Laparoscopic surgery for complicated diverticular disease, including fistulas, has not yet been accepted as the treatment of choice. It is usually reserved for selected cases. Internal fistulas that complicate diverticulitis are usually associated with a high conversion rate and postoperative morbidity due to the presence of dense adhesions. However, in several series, conversion is limited to acceptable rates. It seems that high technical skills could lower conversion rates, as well as intra- and post-operative complications. Other authors report that application of laparoscopy is irrelevant to recurrence rates [7]. Colic resection has high mortality and morbidity rates due to adhesions, paracolic abscesses, colon and mesocolon shortening, and colic parietal thickening, that are caused by diverticulitis [10]. For this reason only a few authors prefer a conservative approach. Naraynsingh et al. reported modified Hinchey classification for surgical decision making in patients with perforated leftsided diverticulitis with faecal peritonitis. They suggested colic resection only in colic scarring and stricture and a possible conservative treatment for colovesical fistula [11]. Lewis and Abercrombie reported the results of 3 cases of colovesical fistula treated with open conservative surgery, removing only the fistulous tract and suturing the parietal defects. The follow-up at 12 and 26 months was successful in two cases. In the third case urinary infection occurred and the patient died 1 year after surgery for bowel obstruction, but no fistula recurrence was evident [12]. Moorthy et al. performed five laparoscopic assisted procedures for colovesical fistulas. The bowel was mobilized intracorporeally and then exteriorized through a small incision. The mesenteric vascular division and bowel resection was carried out extracorporeally in three cases. In 2 cases due to Crohn's disease and diverticulitis, only stapled fistulectomy was performed without colic resection. The patient with colovesical fistula secondary to diverticulitis had a recurrence of the fistula [13]. According to our knowledge, this is the first time colovesical fistula has been treated with totally laparoscopic conservative surgery. Our surgical technique is a laparoscopic approach including dissection and removal of the fistulous tract, curettage of necrotic and inflammatory bladder tissue surrounding the fistula site, suturing and placement of fatty tissue between the colon and bladder. The key point is to carefully prepare the surgical plan between the colon and bladder wall in order to identify the fistulous tract. Identification of the correct cleavage plan allows one to reduce the intra- and post-operative bleeding, the conversion rates and the post-operative morbidity. In our opinion this technique could be performed when the diverticular disease is slight and it is possible to identify and prepare accurately the fistulous tract between the colon and bladder. In our case diverticular disease was slight and inflammation of the colon-sigma was not severe. Indeed the fistula was secondary to polypectomy in a sigmoid diverticulum, where the muscle layer is absent and the wall is weaker. Trecca et al. report that 
the risk of colon perforation after colonoscopy is $0.01-3 \%$. The incidence increases when the colonoscopy is associated with operative procedures such as biopsy or polypectomy [14]. Magdeburg et al. report occurrence of colovesical fistula after colonoscopy in $0.055 \%$ of cases. The incidence increases to $1.24 \%$ when operative colonoscopy is performed [15]. The operative time is comparable to the open approach with colic resection. The postoperative pain was low as proved by the low score on the VAS scale and low requirement of analgesic therapy. There was no incisional hernia during the 4-year follow-up. These results compare favourably with those reported for open surgery [7]. The early restoration of bowel movement due to preserving intestinal integrity and to the laparoscopic approach allows shorter hospital stay and faster postoperative recovery. When necrotic and inflammatory tissue is not evident in the bladder wall, bladder cuff excision is not necessary, but curettage of the fistula site is successful.

\section{Conclusions}

Laparoscopic conservative surgery not including colic resection for colovesical fistula is safe and feasible. Efficacy of this technique is proved by no recurrence and no complications at 4-year follow-up. Larger series are necessary before proposing it as standard treatment. In our opinion laparoscopic conservative surgery may represent a therapeutic option in selected cases, especially if diverticular disease and inflammation are slight.

\section{Acknowledgments}

Written informed consent was obtained from the patient for publication of this case report and any accompanying images. A copy of the written consent is available for review by the Editor-in-Chief of this journal.

\section{References}

1. Nishimori H, Hirata K, Fukui R, et al. Vesico-ileosigmoidal fistula caused by diverticulitis: report of a case and literature review. J Korean Med Sci 2003; 18: 433-6.

2. Pontari MA, McMillen MA, Garvey RH, Ballantyne GH. Diagnosis and treatment of enterovesical fistulae. Am Surg 1992; 58: 258-63.

3. Pironi D, Candioli S, Manigrasso A, et al. Complicated diverticular disease: the colo-vesical fistula. Presentation of three clinical cases and review of the literature. G Chir 2006; 27: 15-20.
4. Salzmann H, Lillie D. Diverticular disease diagnosis and treatment. Am Fam Physician 2005; 72: 1229-34.

5. Reisman Y, Ziv Y, Kravrovitc D, et al. Diverticulitis: the effect of age and location on the course of disease. Int I Colon Rectal Dis 1999; 14: 250-4.

6. Daniels IR, Bekdash B, Scott HJ, et al. Diagnostic lessons learnt from series of enterovesical fistulae. Colorectal Dis 2002; 4: 459-62.

7. Menenakos E, Hahnloser D, Nassiopoulos K, et al. Laparoscopic surgery for fistulas that complicate diverticular disease. Langenbecks Arch Surg 2003; 388: 189-93.

8. Dozois EJ. Operative treatment of recurrent or complicated diverticulitis. J Gastrointest Surg 2008; 12: 1321-3.

9. Comparato G, Pilotto A, Franze A, et al. Diverticular disease in the elderly. Dig Dis 2007; 25: 151-9.

10. Cirocchi R, Farinella E, Trastulli S, et al. Elective sigmoid colectomy for diverticular disease. Laparoscopic vs. open surgery: a systematic review. Colorectal Dis 2012; 14: 671-83.

11. Naraynsingh V, Maharaj R, Hassranah D, et al. Perforated leftsided diverticulitis with faecal peritonitis: is the Hinchey classification the best guide for surgical decision making? Tech Coloproctol 2011; 15: 199-203.

12. Lewis SL, Abercrombie GF. Conservative surgery for vesicocolic fistula. J R Soc Med 1984; 77: 102-4.

13. Moorthy K, Shaul T, Foley RJ. The laparoscopic management of benign bowel fistulas. JSLS 2004; 8: 356-8.

14. Trecca A, Gaj F, Gagliardi G. Our experience with endoscopic repair of large colonoscopic perforations and review of the literature. Tech Coloproctol 2008; 12: 315-22.

15. Magdeburg R, Collet P, Post S, Kaehler G. Endoclipping of iatrogenic colonic perforation to avoid surgery. Surg Endosc 2008; 22: 1500-4.

Received: 3.06.2012, revised: 30.08.2012, accepted: 18.09.2012. 